\title{
Changes in leukocyte subsets of pregnant gilts experimentally infected with porcine reproductive and respiratory syndrome virus and relationships with viral load and fetal outcome
}

\author{
Andrea Ladinig ${ }^{1,4^{*}}$, Wilhelm Gerner ${ }^{2}$, Armin Saalmüller ${ }^{2}$, Joan K Lunney ${ }^{3}$, Carolyn Ashley ${ }^{1}$ and John CS Harding ${ }^{1}$
}

\begin{abstract}
In spite of more than two decades of extensive research, the understanding of porcine reproductive and respiratory syndrome virus (PRRSv) immunity is still incomplete. A PRRSv infection of the late term pregnant female can result in abortions, early farrowings, fetal death, and the birth of weak, congenitally infected piglets. The objectives of the present study were to investigate changes in peripheral blood mononuclear cell populations in third trimester pregnant females infected with type 2 PRRSv (NVSL 97-7895) and to analyze potential relationships with viral load and fetal mortality rate. PRRSv infection caused a massive, acute drop in total leukocyte counts affecting all PBMC populations by two days post infection. Except for B cells, cell counts started to rebound by day six post infection. Our data also show a greater decrease of naïve B cells, T-helper cells and cytolytic T cells than their respective effector or memory counterparts. Absolute numbers of $T$ cells and $\gamma \delta T$ cells were negatively associated with PRRSv RNA concentration in gilt serum over time. Additionally, absolute numbers of T helper cells may be predictive of fetal mortality rate. The preceding three leukocyte populations may therefore be predictive of PRRSV-related pathological outcomes in pregnant gilts. Although many questions regarding the immune responses remain unanswered, these findings provide insight and clues that may help reduce the impact of PRRSv in pregnant gilts.
\end{abstract}

\section{Introduction}

In spite of more than two decades of extensive research, understanding of porcine reproductive and respiratory syndrome virus (PRRSv) immunity is still incomplete. PRRSv is able to persist in infected pigs for several months $[1,2]$ and uses different evasion strategies to circumvent innate and adaptive immune responses, summarized in several reviews [3-5]. Numerous reports have investigated immune responses against PRRSv in vivo including the measurement of cytokine production, the investigation of immune cells, or the measurement of antibody responses. However, a direct comparison of results across different experiments is complicated by several factors including

\footnotetext{
* Correspondence: andrea.ladinig@vetmeduni.ac.at

'Department of Large Animal Clinical Sciences, Western College of Veterinary Medicine, University of Saskatchewan, Saskatoon, SK, Canada

${ }^{4}$ Current address: University Clinic for Swine, Department for Farm Animals and Veterinary Public Health, University of Veterinary Medicine Vienna, Veterinaerplatz 1, 1210 Vienna, Austria

Full list of author information is available at the end of the article
}

the use of different virus isolates, age and genetics of animals, as well as criteria used to measure immune responses. For the investigation of immune cells by flow cytometry (FCM), additional factors complicating interpretation include methods of data presentation (absolute numbers versus percentages of different cell populations) as well as variation in marker selection used to define leukocyte subsets. In addition, investigations of peripheral blood mononuclear cells (PBMC) subsets in response to PRRSv infection have mainly used nursery or growing pigs in PRRSv respiratory models, whereas reports using pregnant females are sparse. Nielsen et al. [6] inoculated sows at 90 days of gestation to investigate leukocyte populations in piglets surviving in utero infection with PRRSv, but did not characterize leukocyte populations in sows. Christianson et al. [7] investigated peripheral blood leukocytes in sows experimentally infected with PRRSv in mid-gestation. A significant decrease in total leukocytes was found at 3 and 7 days post infection (dpi) and was 
most pronounced at $7 \mathrm{dpi}$. Absolute numbers of CD $172 \mathrm{a}^{+}$cells, CD $1^{+}$cells, $\mathrm{CD}^{+}$and $\mathrm{CD} 8 \alpha^{+} \mathrm{T}$ cells were significantly decreased compared to non-infected controls at 3 to $7 \mathrm{dpi}$; cell counts returned to control levels by $14 \mathrm{dpi}$ [7].

As there are few reports describing changes in PBMC populations in late term pregnant sows or gilts following PRRSv infection, and no studies correlated changes in subpopulations with clinical outcome, the objectives of the present study were to: 1) characterize changes in the major PBMC subpopulations (monocytes, NK cells, B and $\mathrm{T}$ cells) of third trimester pregnant gilts following PRRSv infection; 2) analyze phenotypic changes of the major $\mathrm{T}$ cell populations ( $\gamma \delta \mathrm{T}$ cells, $\mathrm{T}$ helper cells and cytolytic T cells (CTL)) following PRRSv infection; 3) investigate relationships between PBMC subpopulations and viral load in gilt serum and tissues; 4) investigate relationships between PBMC subpopulations and fetal mortality rate defined at the level of the gilt as percent dead fetuses per litter.

\section{Materials and methods}

\section{Experimental procedures and sample collection}

The experimental protocol is described in detail in Ladinig et al. [8]. Briefly, on experimental day 0 (0 days post inoculation; dpi), 114 pregnant Landrace gilts (gestation day $85( \pm 1)$ ) split over 12 replicates were inoculated (INOC) with PRRSv isolate NVSL 97-7895 $\left(1 \times 10^{5}\right.$ TCID $_{50} ; 2 \mathrm{~mL}$ intramuscularly and $1 \mathrm{~mL}$ into each nostril), while 19 control gilts were similarly sham inoculated (CTRL). Heparinized blood samples were collected on $0,2,6$, and $19 \mathrm{dpi}$, and sera on 0, 2, 6, and $21 \mathrm{dpi}$. Automated white blood cell (WBC) counts (Z2 Coulter Particle Count and Size Analyzer, Beckman Coulter Inc., FL, USA) and manual differential counts were performed (300 cells total) on heparinized blood samples. On 21 dpi (gestation day $106 \pm 1$ ), gilts were humanely euthanized and necropsied. Fetal preservation status was recorded and the percent dead fetuses were calculated for each litter. Samples of lung, tonsil, reproductive (Lnn. uterini) and tracheobronchial lymph node from each gilt, as well as a sample of the uterus including adherent fetal placental layers adjacent to the umbilical stump of each fetus were collected and immediately frozen at $-80{ }^{\circ} \mathrm{C}$ until further processing. The experiment was approved by the University of Saskatchewan's Animal Research Ethics Board, and adhered to the Canadian Council on Animal Care guidelines for humane animal use (permit \#20110102).

\section{Isolation of PBMC and FCM staining}

PBMC were isolated from whole blood samples by gradient centrifugation using lymphocyte separation medium (Ficoll-Paque $^{\mathrm{Tm}}$ PLUS, GE Healthcare, Mississauga, ON,
Canada). Isolated PBMC were counted and transferred into staining buffer (PBS $+0.2 \%$ gelatin $+0.03 \%$ sodium azide).

For phenotypic analyses of PBMC, 5 sets of marker panels to detect surface antigens were used in two- or three-color labelling. Table 1 summarizes monoclonal antibodies (mAbs) used to characterize different PBMC subsets. Where commercially available, directly conjugated mAbs were used; otherwise, mAbs specific for CD8 $\beta$, swine leukocyte antigen-DR (SLA-DR), and the $\gamma \delta$ T cell receptor (TCR), produced from hybridoma supernatants at the Institute of Immunology, University of Veterinary Medicine Vienna, Austria, were used in combination with fluorochrome-conjugated, isotype-specific secondary Abs (Table 1). Isotype-matched non-specific antibodies were used as negative controls. PBMC staining was performed in U-bottom 96-well microtiter plates $\left(1 \times 10^{6}\right.$ cells per well). All incubations were performed on ice in the dark. Mastermixes of primary Abs were prepared and $30 \mu \mathrm{L}$ was added to each well prior to the first $20 \mathrm{~min}$ incubation step. After two washes in $200 \mu \mathrm{L}$ of staining buffer, mastermixes of secondary Abs $(10 \mu \mathrm{L}$ per well) were added and cells were incubated for another $20 \mathrm{~min}$. Finally, cells were washed twice in staining buffer and fixed by resuspension in $200 \mu \mathrm{L}$ of $2 \%$ formaldehyde solution (PBS $+2 \%$ formaldehyde solution (formaldehyde 37 wt \% solution in water stabilized with 7-8\% methanol, Alfar Aesar, Ward Hill, MA, USA)).

To stain the intracellular epitope recognized by the CD79 $\alpha$-specific mAb HM57, cells were fixed and permeabilized using a commercial kit (BD Cytofix/Cytoperm, BD Biosciences, Mississauga, ON, Canada) according to the manufacturer's instructions.

Prior to incubation and after each wash step, cells were resuspended using a plate shaker. For compensation controls, single-stain samples were prepared for each fluorochrome.

\section{FCM analyses}

Stained cells were analyzed using a FACSCalibur flow cytometer (BD Biosciences, Mississauga, ON) equipped with 2 lasers (488 and $635 \mathrm{~nm}$ ). At least $5 \times 10^{4}$ cells were collected per sample. Results were analyzed using FlowJo, version 7.6.5 (Tree Star, Inc., Ashland, Oregon, USA). Gates were set according to isotype controls and fluorescence minus one (FMO) control samples [9]. The same gate position was used for all samples with a particular marker combination. Cell numbers for each population were corrected by subtracting the number of cells stained by isotype-matched non-specific antibodies. Automated WBC counts and manual differential counts (total number of lymphocytes plus total number of monocytes) were used to calculate the absolute numbers of different PBMC subsets. 
Table 1 Antibodies used for flow cytometry analyses

\begin{tabular}{|c|c|c|c|c|c|}
\hline Antigen & Clone & Isotype & Source & Fluorochrome & Secondary Ab \\
\hline \multicolumn{6}{|c|}{ T-helper cells } \\
\hline CD3 & BB23-8E6-8C8 & $\lg G 2 a$ & BD Biosciences & PerCP-Cy5.5 & \\
\hline CD4 & $74-12-4$ & $\lg G 2 b$ & BD Biosciences & FITC & \\
\hline CD8a & $76-2-11$ & $\lg G 2 a$ & BD Biosciences & Alexa647 & \\
\hline \multicolumn{6}{|c|}{ Cytolytic $T$ cells } \\
\hline CD3 & BB23-8E6 & $\lg G 2 b$ & Southern Biotech & FITC & \\
\hline $\operatorname{CD} 8 \beta$ & PPT23 & $\lg G 1$ & In-house & & goat anti-mouse lgG1, Alexa647 (Invitrogen) \\
\hline SLA-DR & MSA3 & $\lg G 2 a$ & In-house & & goat anti-mouse lgG2a, PE (Southern Biotech) \\
\hline \multicolumn{6}{|l|}{$\gamma \delta T$ cells } \\
\hline Pan- $-\gamma \delta$ & PPT16 & $\lg G 2 b$ & In-house & & goat anti-mouse lgG2b, Alexa488 (Invitrogen) \\
\hline CD2 & RPA-2.10 & $\lg G 1$ & AbD Serotec & PE & \\
\hline CD8a & $76-2-11$ & $\lg G 2 a$ & BD Biosciences & Alexa647 & \\
\hline \multicolumn{6}{|l|}{ NK cells } \\
\hline CD3 & BB23-8E6-8C8 & $\lg G 2 a$ & BD Biosciences & PerCP-Cy5.5 & \\
\hline CD8a & $76-2-11$ & $\lg G 2 a$ & BD Biosciences & Alexa647 & \\
\hline \multicolumn{6}{|l|}{ B cells } \\
\hline CD21 & B-ly4 & $\lg G 1$ & $\mathrm{BD}$ & APC & \\
\hline CD79a & HM57 & $\lg G 1$ & Dako & PE & \\
\hline \multicolumn{6}{|c|}{ Monocytes } \\
\hline CD172a & $74-22-15$ & $\lg G 1$ & Southern Biotech & PE & \\
\hline CD4 & $74-12-4$ & $\lg G 2 b$ & BD Biosciences & Alexa647 & \\
\hline CD14 & MIL-2 & $\lg G 2 b$ & AbD Serotec & FITC & \\
\hline
\end{tabular}

\section{Quantification of PRRSv RNA}

PRRSv RNA concentrations were measured in gilt serum collected on $0,2,6$ and 21 dpi (target $\log _{10}$ copies $/ \mu \mathrm{L}$ ), and in tissues collected at termination (21 dpi) (target $\log _{10}$ copies/mg), by strain-specific in-house quantitative reverse transcription polymerase chain reaction (qRTPCR) as previously described [8].

\section{Statistical analysis}

Separate statistical analyses were conducted using Stata 13 (STATA Corp, College Station, Texas, USA) to address each of the objectives. To meet the key model assumptions, data were log-transformed (log base $e_{10}$ ) as appropriate. Firstly, to determine if major PBMC subpopulations, including major $\mathrm{T}$ cell populations, differed between INOC and CTRL gilts over time, multilevel mixed-effects linear regression models were developed. These models used gilt as a random effect and accounted for repeated measures by day. All remaining analyses used data from INOC gilts only. Secondly, potential relationships between PBMC subsets (major PBMC subpopulations and major $\mathrm{T}$ cell populations) and PRRS viral load in serum and tissues were analyzed. For these analyses, multilevel mixed-effects linear regression models controlling for experimental replicate were used. Area under the curve (AUC) from 0 to 19/21 dpi was calculated for PRRSv RNA concentration in serum (target copies $/ \mu \mathrm{L}$ ) and for the total number of each PBMC subset (cells $\times 10^{9} /$ liter) using the formula AUC $=\left(\mathrm{t}_{1}-\mathrm{t}_{0}\right)\left(\mathrm{a}_{1}+\mathrm{a}_{0}\right) / 2+\left(\mathrm{t}_{2}-\mathrm{t}_{1}\right)\left(\mathrm{a}_{1}+\right.$ $\left.a_{2}\right) / 2+\ldots+\left(t_{n}-t_{n-1}\right)\left(a_{n-1}+a_{n}\right) / 2$. The final objective, to determine potential associations between the AUC of PBMC subsets and fetal mortality rate (represented as the percentage of dead fetuses per litter), was analysed using multilevel mixed-effects linear regression models controlling for experimental replicate. To account for multiple comparisons, all associations were considered statistically significant if $P<0.01$. All final models were evaluated to ensure normality and homoscedasticity of residuals.

\section{Results}

Changes in total leukocytes and major PBMC subpopulations in response to PRRSv infection

One gilt died (11 dpi) and two gilts aborted (17 dpi, 20 dpi) after PRRSv inoculation; results from those three gilts were excluded from further analysis. Thus, data is 
presented on 111 INOC and 19 CTRL gilts. With the exception of reduced feed intake and increased rectal temperatures in individual gilts, no severe clinical signs were observed following infection.

Changes in total leukocyte numbers in INOC and CTRL gilts following PRRSv infection are displayed in Figure 1. Total leukocyte numbers in INOC gilts decreased $45 \%$ from $0 \mathrm{dpi}\left(11.0 \pm 1.8 \times 10^{9} / \mathrm{L}\right)$ to $2 \mathrm{dpi}\left(6.1 \pm 2.3 \times 10^{9} / \mathrm{L}\right)$ $(P<0.001)$. Values returned to pre-inoculation levels on 19 dpi. Neutrophil counts (data not displayed in figures) were also significantly decreased in INOC compared to CTRL on 6 dpi (INOC: $2.3 \pm 0.9 \times 10^{9} / \mathrm{L}, \mathrm{CTRL}: 3.2 \pm 1.3 \times 10^{9} / \mathrm{L}$, $P=0.002$ ) and trended to increase on $19 \mathrm{dpi}$ (INOC: $4.1 \pm$ $2.0 \times 10^{9} / \mathrm{L}$, CTRL: $\left.3.0 \pm 1.6 \times 10^{9} / \mathrm{L}, P=0.018\right)$.

Monocytes were identified by a CD172 ${ }^{\text {high }} \mathrm{CD} 4^{-} \mathrm{CD} 14^{+}$ phenotype (Figure 2A). Total numbers were significantly decreased in INOC gilts compared to CTRL on $2 \mathrm{dpi}$ $(P<0.001)$ and $6 \mathrm{dpi}(P=008)$ (Figure $2 \mathrm{~B})$. Since there was a significant increase in the percentage of monocytes within PBMC from INOC gilts on D2 (Figure 2C), the drop in absolute numbers was less severe compared to other PBMC subpopulations (see below). Absolute numbers of monocytes on 19 dpi trended to increase in INOC compared to CTRL gilts $(P=0.045)$ (Figure $2 \mathrm{~B})$.

$\mathrm{NK}$ cells were identified by a $\mathrm{CD} 3^{-} \mathrm{CD} 8 \alpha^{+}$phenotype (Figure $3 \mathrm{~A}$ ). Their absolute numbers were highly variable in both INOC and CTRL groups. Compared to CTRL gilts, INOC gilts had significantly greater NK cells numbers on 0 dpi $(P=0.005)$, then showed a significant decrease on $2 \mathrm{dpi}(P<0.001)$ and $6 \mathrm{dpi}(P=0.009)$ followed by an increase on $19 \mathrm{dpi}$ that trended towards significance $(P=0.015)$ (Figure 3B). The drop in NK cells from $0 \mathrm{dpi}$

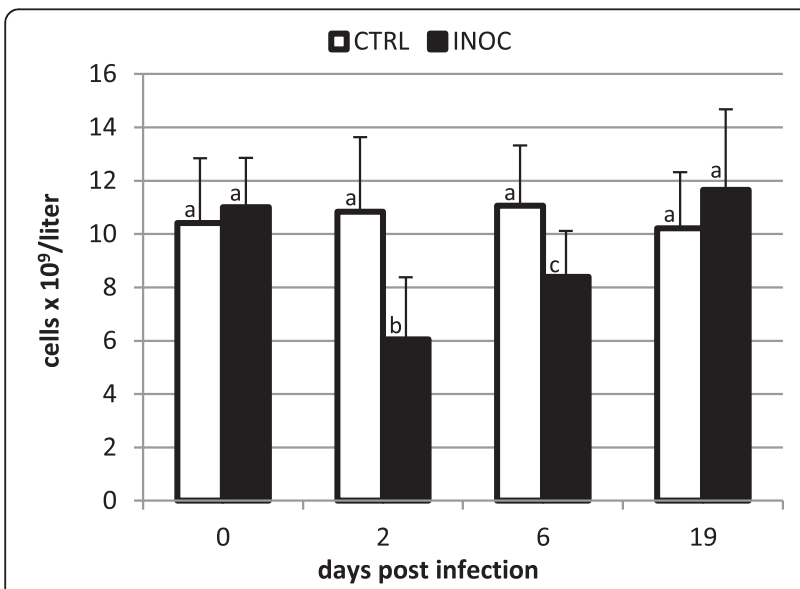

Figure 1 Changes in total leukocyte counts in response to PRRSv infection in pregnant gilts. Mean (+SD) total leukocyte counts are presented from 111 INOC and 19 CTRL gilts for the respective study days. Superscript letters indicate significant differences $(P<0.001)$ between INOC and CTRL gilts and between study days within each treatment group. $\left(0.22 \pm 0.1 \times 10^{9} / \mathrm{L}\right)$ to $2 \mathrm{dpi}\left(0.05 \pm 0.04 \times 10^{9} / \mathrm{L}\right)$ represented a $73 \%$ decrease compared to $0 \mathrm{dpi}$ counts, the most prominent drop of any major PBMC subpopulation.

Total B cells were identified by a CD79 $\alpha^{+}$phenotype (Figure 4A, turquois gate). Their absolute counts also decreased significantly on $2 \mathrm{dpi}$ and $6 \mathrm{dpi}(P<0.001)$ in INOC gilts compared to CTRL (Figure 4B). In contrast to the remaining PBMC subpopulations, total $\mathrm{B}$ cells did not rebound on $6 \mathrm{dpi}$, but rather continued to decrease when compared to 2 dpi $\left(0.57 \pm 0.2 \times 10^{9} / \mathrm{L}\right.$ on $2 \mathrm{dpi}$; $0.66 \pm 0.3 \times 10^{9} / \mathrm{L}$ on $6 \mathrm{dpi}$ ), before rebounding to preinoculation values on $19 \mathrm{dpi}$. CD21-defined B cell subpopulations, which can be used to distinguish naïve and activated from effector and memory B cells [10], were also studied (Figure 4A, purple and pink gates). Both $\mathrm{B}$ cell subpopulations, $\mathrm{CD} 21^{-} \mathrm{CD} 79 \alpha^{+}$(Figure 4C) and CD $21^{+}$CD79 $\alpha^{+}$(Figure 4D), were affected. CD $21^{+}$B cells decreased more rapidly and severely than CD $21^{-} \mathrm{B}$ cells, with their absolute numbers dropping to $35 \%$ of 0 dpi values by 2 dpi (Figure 4D). By contrast, the CD21- B cell subpopulation in INOC gilts decreased to only $91 \%$ and $70 \%$ of $0 \mathrm{dpi}$ counts by $2 \mathrm{dpi}$ and $6 \mathrm{dpi}$ respectively, and trended higher than CTRL on $19 \mathrm{dpi}(P=0.031)$ (Figure $4 C$ ). In INOC gilts, the percentage of B cells expressing CD21 was significantly lower at all time points after infection compared to 0 dpi (Figure 4E).

Total $\mathrm{T}$ cells were identified by a $\mathrm{CD}^{+}$phenotype (Figure 5A). Their absolute numbers decreased significantly in INOC compared to CTRL gilts on 2 dpi $(P<0.001)$, and trended lower on $6 \mathrm{dpi}(P=0.013)$ (Figure 5B). Three subpopulations of $\mathrm{T}$ cells, namely $\gamma \delta \mathrm{T}$ cells, $\mathrm{T}$ helper cells, and cytolytic T cells (CTLs), were analyzed in more detail.

\section{$\gamma \delta$ T cells}

$\gamma \delta$ T cells, identified by a mAb recognizing a CD3 molecule associated with the TCR- $\gamma \delta$ (clone PPT16) [11], were analyzed for the expression of CD2 and CD8 $\alpha$. This lead to the identification of three distinct phenotypes: $\mathrm{CD} 2^{-} \mathrm{CD} 8 \alpha^{-}$, $\mathrm{CD} 2{ }^{-} \mathrm{CD} 8 \alpha^{+}$, and $\mathrm{CD} 2^{+} \mathrm{CD} 8 \alpha^{+} \gamma \delta \mathrm{T}$ cells (Figure $6 \mathrm{~A}$ ). Absolute numbers of $\gamma \delta$ T cells dropped 45\% from 0 to 2 dpi in INOC gilts; 2 dpi counts were significantly lower than in CTRL gilts $(P<0.001)$ (Figure 6B). All three subpopulations showed a significant decrease on 2 dpi compared to CTRL gilts $(P<0.001)$ (Figure 6C to $\mathrm{E})$. While absolute numbers of $\mathrm{CD}^{-} \mathrm{CD} 8 \alpha^{-} \gamma \delta \mathrm{T}$ (Figure 6C) and $\mathrm{CD} 2^{-} \mathrm{CD} 8 \alpha^{+}$(Figure 6D) $\gamma \delta$ T cells did not differ significantly between INOC and CTRL on 6 and $19 \mathrm{dpi}, \mathrm{CD} 2^{+} \mathrm{CD} 8 \alpha^{+} \gamma \delta \mathrm{T}$ cells (Figure 6E) were significantly lower in INOC on $6 \mathrm{dpi}(P<0.001)$ and also trended lower on $19 \mathrm{dpi}(P=0.028)$. While the percentage of the $\mathrm{CD} 2^{-} \mathrm{CD} 8 \alpha^{-}$phenotype increased significantly in INOC gilts after infection (Figure 6F), the percentages of both $\mathrm{CD}_{2}^{-} \mathrm{CD} 8 \alpha^{+}$(Figure $6 \mathrm{G}$ ) and $\mathrm{CD} 2^{+} \mathrm{CD} 8 \alpha^{+}$ 


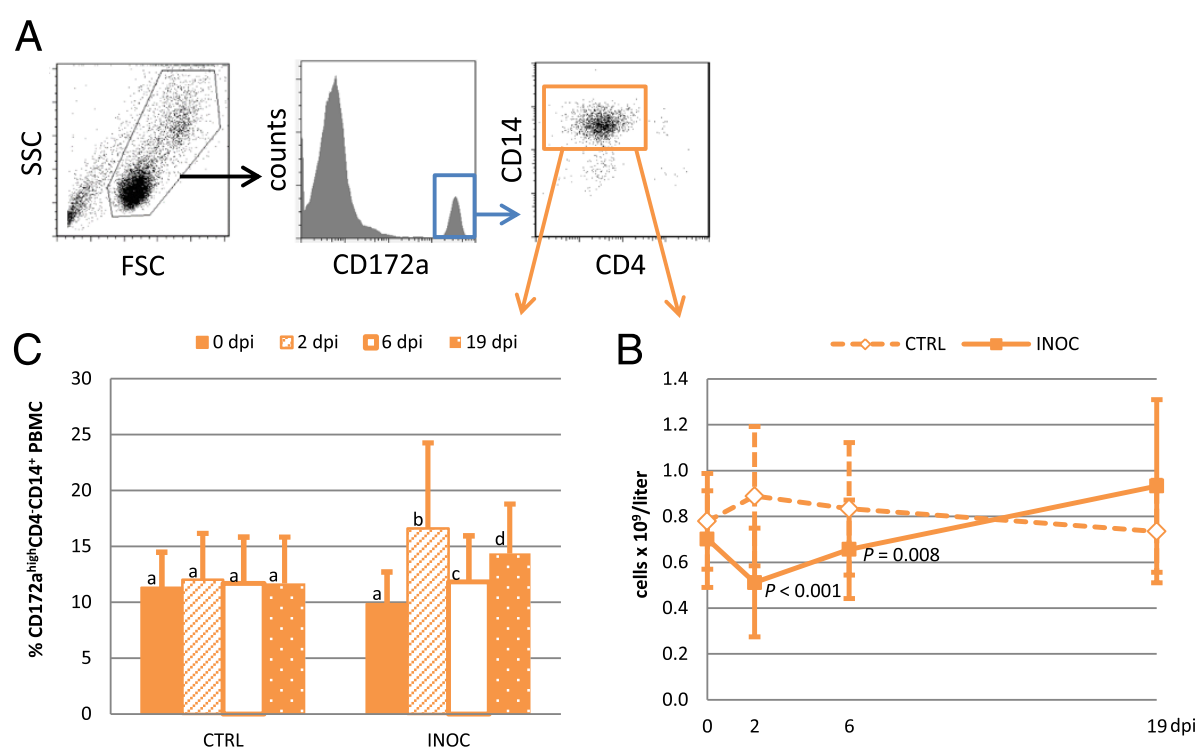

Figure 2 Changes in monocytes in response to PRRSv infection in pregnant gilts. A) Dot plots: The gating strategy for monocytes $\left(\mathrm{CD} 172 \mathrm{a}^{\text {high }} \mathrm{CD} 4^{-} \mathrm{CD} 14^{+}\right)$is demonstrated using representative data from gilt \#53. B) Line chart: Changes in absolute numbers (mean $\pm \mathrm{SD}$ ) of monocytes (orange) are presented from 111 INOC and 19 CTRL gilts over time. P-values indicate significant differences between INOC and CTRL gilts on individual days. C) Bar chart: The mean percentages (+SD) of CD172a high $C D 4^{-} C D 14^{+}$PBMC from INOC and CTRL gilts are presented for the respective study days. Superscript letters indicate significant differences $(P<0.01)$ between study days within INOC or CTRL gilts.

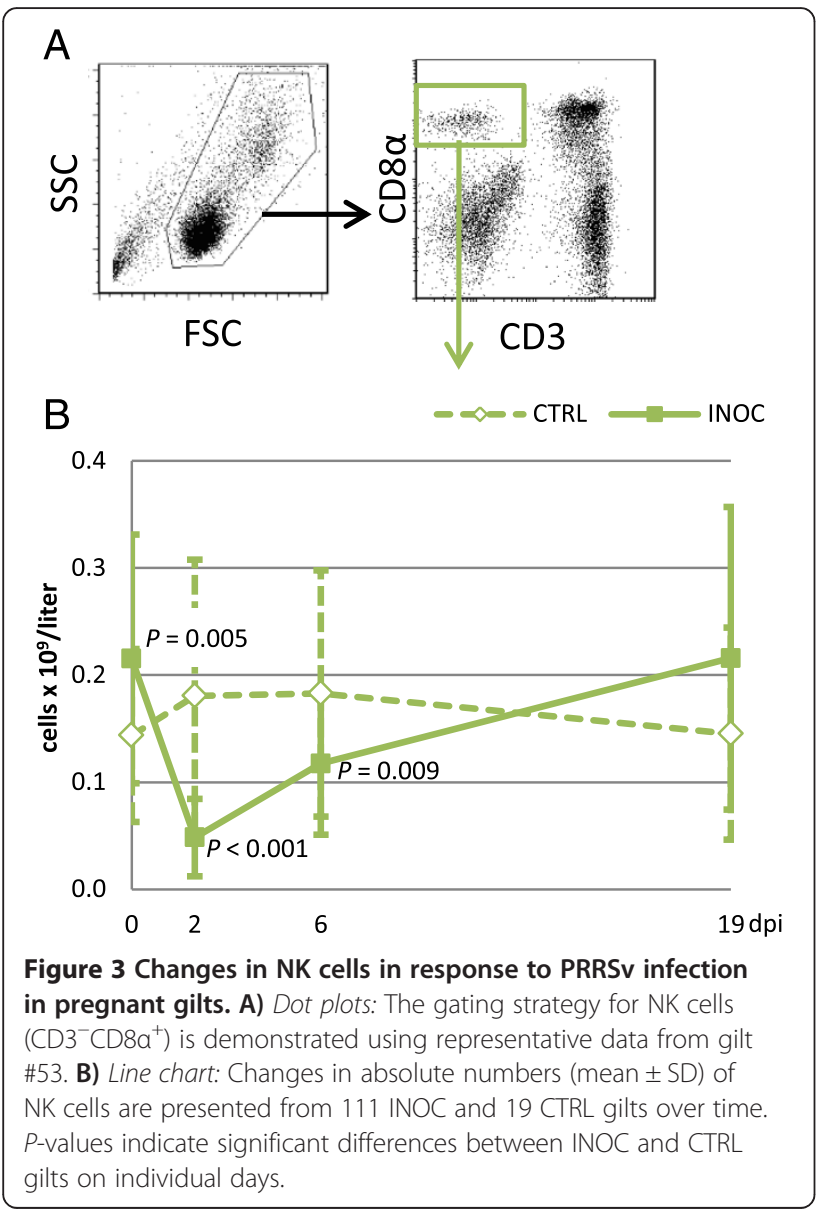

(Figure $6 \mathrm{H}) \gamma \delta \mathrm{T}$ cells were significantly decreased in INOC gilts.

\section{T helper cells}

Absolute $\mathrm{T}$ helper cells, identified by a $\mathrm{CD} 3^{+} \mathrm{CD} 4^{+}$ phenotype (Figure $7 \mathrm{~A}$, yellow gate), were significantly decreased in INOC gilts on $2 \mathrm{dpi}(P<0.001)$ and trended lower on $6 \mathrm{dpi}(P=0.044)$ compared to CTRL (Figure 7B). To investigate their activation/memory status [11], expression of CD8 $\alpha$ was analyzed (Figure 7A, brown and light blue gates). Compared to CRTL, INOC gilts had numerically increased numbers of $\mathrm{CD} 8 \alpha^{-} \mathrm{T}$ helper cells on $0 \mathrm{dpi}$ $(P=0.033)$, but numbers decreased significantly on 2 dpi $(P<0.001)$ (Figure $7 \mathrm{C})$. The decrease in $\mathrm{CD}^{-} \alpha^{-}$ $\mathrm{T}$ helper cells in INOC gilts was substantial with counts dropping to $22 \%\left(0.22 \pm 0.1 \times 10^{9} / \mathrm{L}\right)$ of 0 dpi counts. Absolute numbers of $\mathrm{CD} 8 \alpha^{+} \mathrm{T}$ helper cells were significantly lower in INOC gilts on 2 dpi $(P<0.001)$ and 6 dpi $(P=0.003)$, and trended lower on $19 \mathrm{dpi}(P=0.021)$ compared to CTRL gilts (Figure 7D). However, given the percentage of $\mathrm{T}$ helper cells expressing CD8 $\alpha$ increased significantly on 2 dpi (Figure 7E), the drop in total $\mathrm{T}$ helper cells on $2 \mathrm{dpi}$ to $42 \%$ of the 0 dpi counts was over represented by the $\mathrm{CD} 8 \alpha^{-}$phenotype, rather than the $\mathrm{CD} 8 \alpha^{+}$phenotype.

CTLS

Absolute numbers of CTLs, defined as $\mathrm{CD}^{+} \mathrm{CD} 8 \beta^{+}$ (Figure 8A, blue gate), were significantly decreased in 

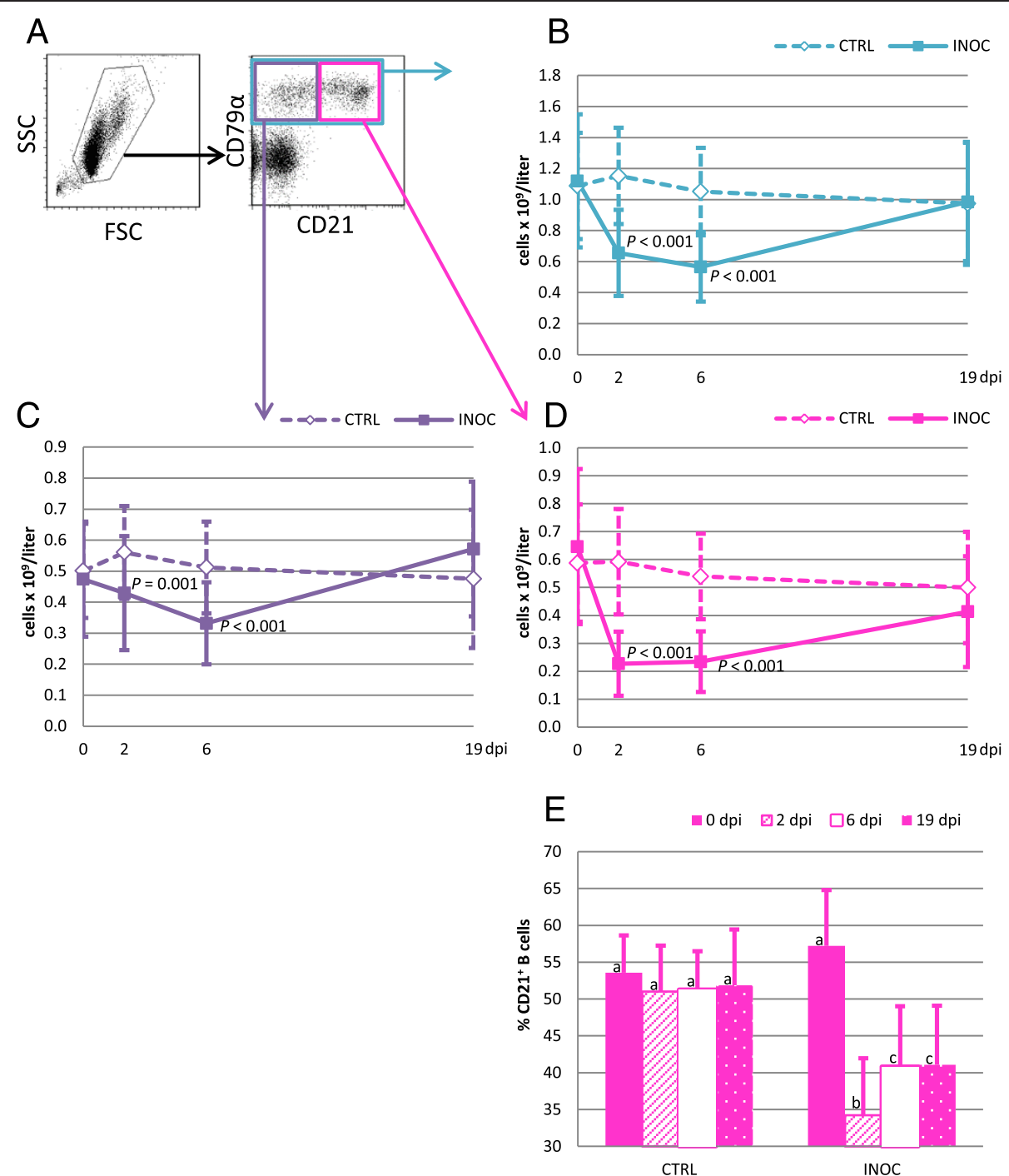

Figure 4 Changes in B cells in response to PRRSv infection in pregnant gilts. A) Dot plots: The gating strategy for B cells $\left(C D 79 a^{+}\right)$and CD21-defined subpopulations is demonstrated using representative data from gilt \#53. B-D) Line charts: Changes in absolute numbers (mean \pm SD) of total B cells (turquois), CD21- (purple), and CD21 (pink) B cells are presented from 111 INOC and 19 CTRL gilts over time. $P$-values indicate significant differences between INOC and CTRL gilts on individual days. E) Bar chart: The mean percentages (+SD) of B cells expressing CD21 within total CD79a $a^{+}$cells from INOC and CTRL gilts are presented for the respective study days. Superscript letters indicate significant differences $(P<0.01)$ between study days within INOC or CTRL gilts.

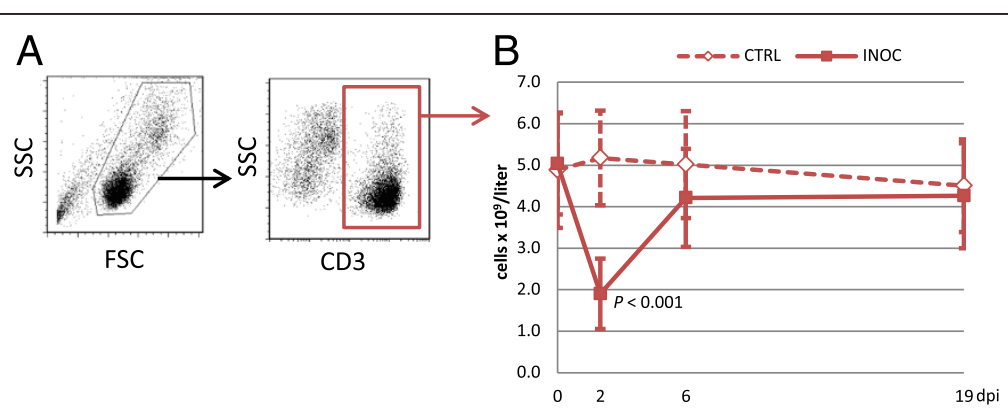

Figure 5 Changes in T cells in response to PRRSv infection in pregnant gilts. A) Dot plots: The gating strategy for T cells (CD $\left.3^{+}\right)$is demonstrated using representative data from gilt \#53. B) Line chart: Changes in absolute numbers (mean \pm SD) of T cells are presented from 111 INOC and 19 CTRL gilts over time. $P$-values indicate significant differences between INOC and CTRL gilts on individual days. 


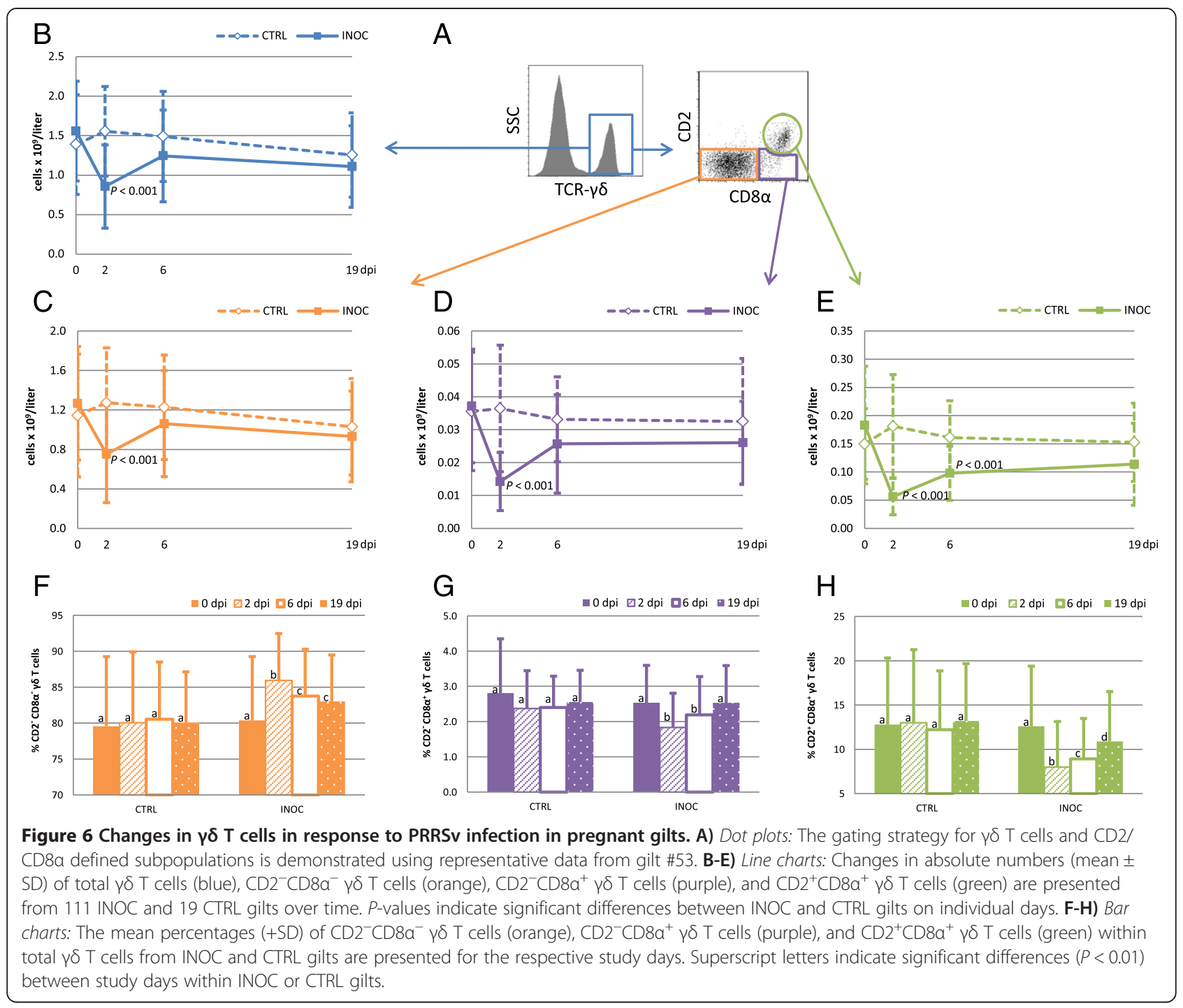

INOC gilts compared to CTRL on 2 dpi $(P<0.001)$ and trended lower on $6 \mathrm{dpi}(P=0.013)$ (Figure 8B). 2 dpi CTL counts in INOC gilts were $77 \%$ lower $(0.30 \pm 0.2 \times$ $10^{9} / \mathrm{L}$ ) compared to $0 \mathrm{dpi}$ counts. CTLs were further analyzed for the expression of SLA-DR, suggested as a potential marker for activation and antigen encounter [11]. Absolute numbers of SLA-DR ${ }^{+}$CTLs showed a similar trend and were significantly lower in INOC gilts on 2 dpi $(P<0.001)$ (Figure $8 C)$. Although the percentage of CTLs expressing SLA-DR did not significantly change in CTRL gilts, in INOC gilts the percentage increased significantly to varying degrees on each study day following PRRS infection (Figure 8D). When analyzing the mean fluorescence intensity (MFI) of the three surface markers identified on CTLs, no changes were observed for CD3 (data not shown). The MFI of CD8 $\beta$ decreased significantly from 0 to $2 \mathrm{dpi}$ in both INOC and CTRL gilts $(P<0.001)$, but the drop was more distinct in INOC
(Figure $8 \mathrm{E}$ ). On the other hand, the MFI of SLA-DR significantly increased in INOC gilts on 2 and 6 dpi (Figure 8F), but decreased in CTRL.

\section{Associations between PBMC subpopulations and PRRS outcome}

Detailed results on viral loads (gilt serum and tissues) and on fetal preservation can be found in Ladinig et al. [8]. Briefly, all INOC gilts were viremic on 2 and $6 \mathrm{dpi}$, and $94 / 111$ (84.7\%) remained viremic until termination. The percentages of tissues that tested positive by PRRSv qRT-PCR at termination (21 dpi) in INOC gilts were 90 , 99, 99, and 100 for lung, tonsil, tracheobronchial lymph node and reproductive lymph node, respectively. The mean viral loads of positive samples were $3.5 \pm 1.2 \log _{10}$ RNA copies/mg in lung, $5.6 \pm 0.8 \log _{10}$ RNA copies/mg in tonsil, $5.8 \pm 0.8 \log _{10}$ RNA copies/mg in reproductive lymph node, and $4.8 \pm 0.9 \log _{10}$ RNA copies/mg in 

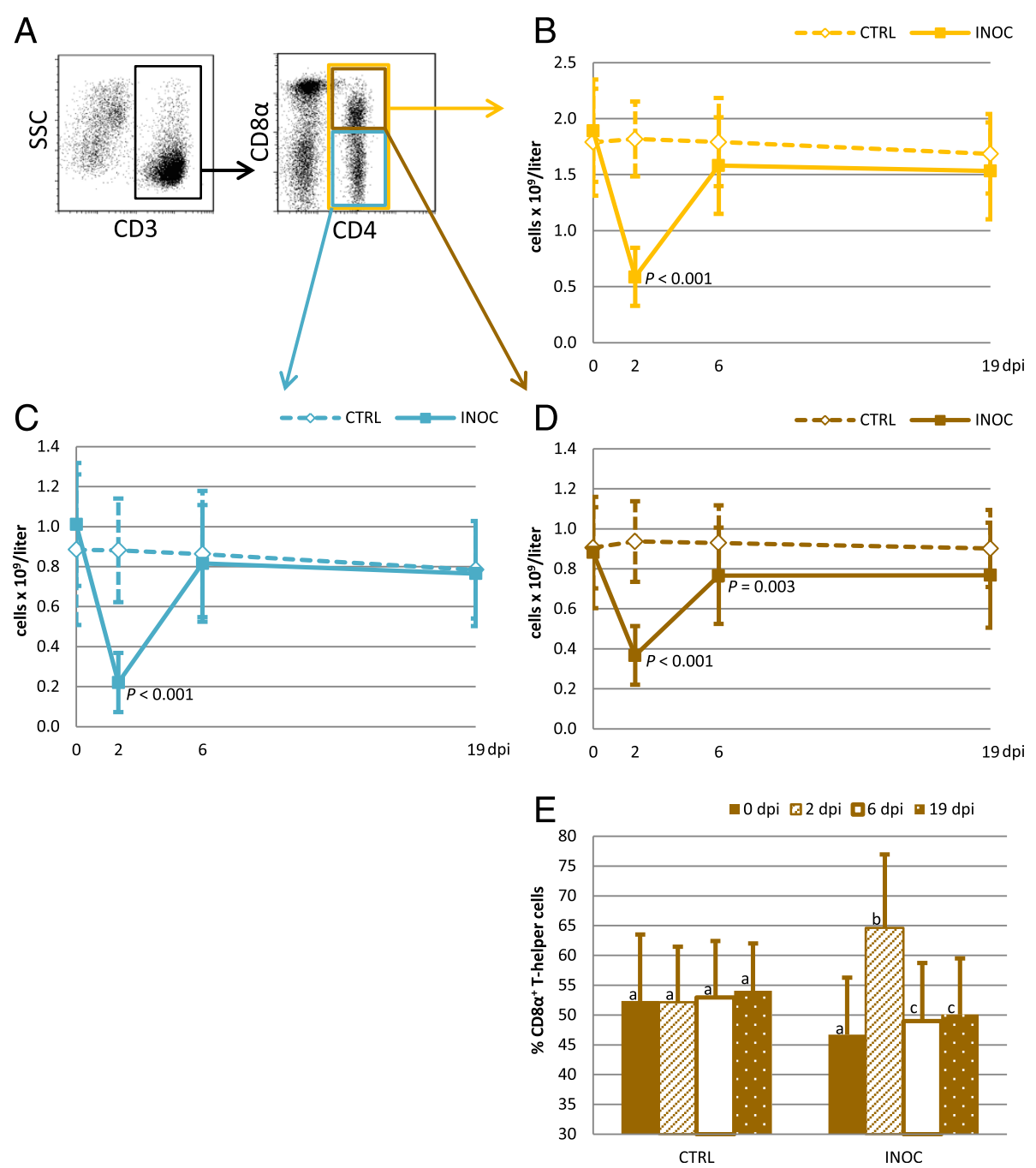

Figure 7 Changes in T helper cells in response to PRRSv infection in pregnant gilts. A) Dot plots: The gating strategy for Thelper cells $\left(\mathrm{CD}^{+}\right)$and $\mathrm{CD} 8 \mathrm{a}$-defined subpopulations is demonstrated using representative data from gilt \#53. B-D) Line charts: Changes in absolute numbers (mean \pm SD) of total T helper cells (yellow), CD8 $a^{-}$T helper cells (light blue), and CD8a ${ }^{+}$T helper cells (brown) are presented from 111 INOC and 19 CTRL gilts over time. P-values indicate significant differences between INOC and CTRL gilts on individual days. E) Bar chart: The mean percentages (+SD) of T helper cells expressing CD8a within total CD4 ${ }^{+} \mathrm{T}$ cells from INOC and CTRL gilts are presented for the respective study days. Superscript letters indicate significant differences $(P<0.01)$ between study days within INOC or CTRL gilts.

tracheobronchial lymph node. In INOC gilts, the percentage of dead fetuses ranged from $0 \%$ to $94.4 \%$ (mean $41.0 \pm 22.8 \%)$.

The total numbers (AUC) from 0 to 19 for monocytes, NK cells, B cells, total T cells, $\gamma \delta \mathrm{T}$ cells, $\mathrm{T}$ helper cells, and CTLs were used to test possible associations with viral load (AUC) and fetal mortality rate. None of the cell subsets were associated with viral load in any gilt tissue. Viral load (AUC) in gilt serum was negatively associated with total T cells $(\beta=-0.140, P=0.007)$ and total $\gamma \delta$ T cells $(\beta=-0.236, P=0.009)$ over time. None of the analyzed PBMC populations were significantly associated with the percentage of dead fetuses per litter. Total $\mathrm{T}$ helper cells (AUC) however, trended to be negatively associated with fetal mortality rate $(\beta=-0.743, P=0.048)$.

\section{Discussion}

The results presented are part of an extensive and complex dataset obtained in a large scale, multi-institutional project aimed at finding phenotypic and genotypic predictors of PRRSv resistance in pregnant gilts. To investigate genotypic variation in the severity of reproductive PRRS, a large number of gilts were experimentally inoculated in the third trimester of gestation. The number of CTRL gilts was reduced to the minimum to provide baseline data. The objectives of this present study were 


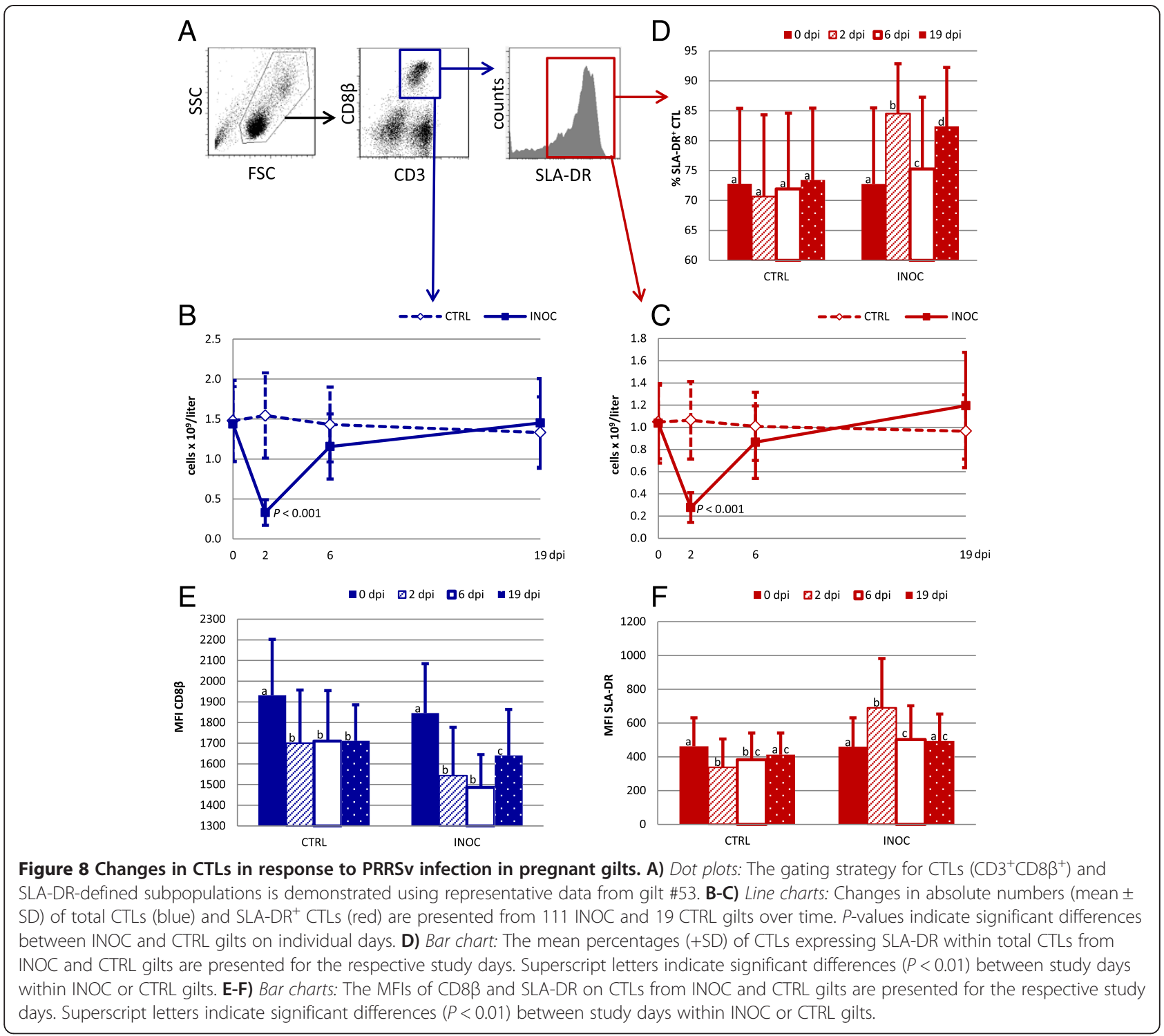

to investigate temporal changes in the major $\mathrm{PBMC}$ and $\mathrm{T}$ cell populations and to determine possible associations with measures of PRRS outcome in a reproductive model.

A massive drop in total leukocyte counts was detected early after infection ( $2 \mathrm{dpi}$ ) in all INOC gilts to varying degrees. Leukocyte counts in INOC gilts started to rebound by 6 dpi. Leukopenia has been demonstrated in several other swine viral infections including African swine fever virus (ASFv) [12], classical swine fever virus (CSFv) [13], pseudorabies virus (PRv) [14], and porcine circovirus type 2 (PCV2) [15]. In both ASF and CSF the leukopenia which mainly involved lymphocytes, occurred during the first week after infection and was associated with necrosis and apoptosis of cells $[12,13]$. Similarly, a significant drop in lymphocytes between 3 and $7 \mathrm{dpi}$ and probably due to apoptosis, was found in pigs infected with H1N2 swine influenza virus. However, the lymphopenia was not accompanied by a drop in total leukocytes [16]. On the other hand, acute PRv infection induced leukopenia involved the loss of up to $40 \%$ of monocytes and up to $50 \%$ of lymphocytes; the authors hypothesized that besides the killing of infected cells, trafficking of effector cells from the circulation to sites of local infection also contributed to the observed leukopenia [14].

The specific mechanisms associated with the leukopenia observed in this study are speculative and cannot be answered with the current dataset; still this study provides several insightful findings. PRRSv induced apoptosis and necrosis of PRRSv-infected and non-infected bystander cells has previously been reported [17-21]. Cells affected by PRRSv induced apoptosis were mainly macrophages and mononuclear cells, as well as epithelial cells $[17,20]$. 
Recently, it was demonstrated that apoptotic cells in thymuses of PRRSv-infected fetuses were predominantly $\mathrm{CD}^{+}$ T cells [22]. Although apoptosis and necrosis might be one possible explanation for the observed leukopenia, in most studies apoptosis and necrosis were observed after 6 dpi $[17,20,23]$. Moreover, PRRSv was reported to activate antiapoptotic pathways in macrophages early in in vitro infection models, while PRRSv-infected macrophages die by apoptosis late in infection [21]. For these reasons and given the acuteness of the response and rebound, the authors believe that apoptosis or necrosis of PBMC is unlikely. Moreover, there are no reports in the literature providing evidence of PRRSv replication within lymphocytes.

Therefore, another possible explanation for the leukopenia detected in the present study is an altered trafficking pattern of leukocyte subsets. Interestingly, the current immunological paradigm is that only effector and effector memory cells traffic into non-lymphoid tissue [24]. However, it also is conceivable that PRRSv infection affects entry and exit of lymphocytes from affected lymph nodes as was shown by early experiments in sheep treated with model antigens $[25,26]$. Obviously, sites of leukocyte migration in our infection model are currently speculative and need to be investigated in future experiments.

The primary sites of PRRSv replication are lung and lymphoid tissues [27]. PRRSv has a tropism to macrophages expressing the receptors sialoadhesin $(\mathrm{Sn})$ and CD163 $[28,29]$. In the present study, monocytes significantly decreased in INOC gilts on 2 and 6 dpi. However, the drop in absolute numbers was less severe compared to other PBMC subsets. Similar to our findings, Dwivedi et al. [30] reported the frequency of $\mathrm{CD} 172 \mathrm{a}^{+}$cells in PBMC to be significantly decreased 2 days after infection of 7 week-old piglets. A more recent study determined that changes in the absolute numbers of monocytes in PRRSv infected, 6 week-old piglets were virus strain dependent, particularly in the first 2 weeks post-infection. However, at 21 dpi piglets infected with all strains of PRRSv showed significantly increased numbers of monocytes compared to uninfected controls [31]. In the present study, a similar trend of increased monocytes on 19 dpi was observed in INOC gilts.

A prominent drop in NK cells was measured in inoculated gilts early after PRRSv infection. As members of the innate immune system, NK cells possess germ-line encoded, invariant receptors recognizing molecules on the surface of infected or malignantly transformed cells. This makes them particularly important in the early phase of viral infections [32,33]. In contrast to our findings, absolute numbers of NK cells were significantly increased at 2 dpi in 7 week-old piglets in direct contact with pen mates infected with type 2 PRRSv (strain MN 1-18-2). In the same experiment, NK cell numbers were not significantly increased in inoculated piglets [30]. An increase in absolute NK cell numbers was also detected at 10 and 35 dpi in 6 week-old piglets infected with certain European PRRSv isolates [31]. In contrast to European subtype 1 strains, the subtype 3 strain Lena did not induce an increase in NK cell numbers until 35 dpi, rather showing numerically decreased NK cell numbers until $10 \mathrm{dpi}$. This agrees with our findings. The differences in NK responses among these experiments might be caused by the different virus isolates used, since the results of Weesendorp et al. [31] clearly demonstrated that absolute numbers of NK cells vary between pigs infected with different virus strains.

We detected no association between viral load in gilt tissues and absolute numbers of major PBMC subpopulations or major $\mathrm{T}$ cell populations over time. However, increased absolute $\mathrm{T}$ cell and $\gamma \delta \mathrm{T}$ cell counts over time were associated with decreased viral load in gilt serum. This may be relevant for the control of PRRS by selection strategies for breeding programs.

B cells, T helper cells and CTLs with an effector or memory phenotype $\left(\mathrm{CD} 21^{-}, \mathrm{CD} 8 \alpha^{+}, \mathrm{SLA}-\mathrm{DR}{ }^{+}\right.$, respectively) were less affected by the drop in absolute counts after PRRSv infection. Studies investigating B cell responses towards PRRSv infection mainly measured $\mathrm{Ab}$ responses in serum of infected pigs. Only a few reports investigated B cells by FCM; results are somewhat difficult to compare due to differences in marker selection used to define B cells. Nevertheless, Christianson et al. [7] found absolute numbers of $\mathrm{CD}^{+}$lymphocytes (which represent about $70 \%$ of $C D 79 \alpha^{+}$B cells; Gerner, unpublished findings) significantly decreased in PRRSv inoculated, mid-gestation sows $7 \mathrm{dpi}$, which is in accordance with our findings.

Similar to $\mathrm{CD} 21^{-}$B cells, total numbers of $\mathrm{CD} 44^{+} \mathrm{CD} 8 \alpha^{+}$ $\mathrm{T}$ helper cells dropped less severely than $\mathrm{CD} 4^{+} \mathrm{CD} 8 \alpha^{-} \mathrm{T}$ helper cells in INOC gilts on $2 \mathrm{dpi}$ after PRRSv infection. It is well known that many porcine $\mathrm{CD} 4^{+} \mathrm{T}$ helper cells in peripheral blood express CD8 $\alpha$ [34] and that both activated and memory $\mathrm{T}$ helper cells belong to the $\mathrm{CD} 4{ }^{+} \mathrm{CD} 8 \alpha^{+}$ population $[35,36]$. This may indicate stronger recruitment of naïve $\mathrm{T}$ helper cells from blood to the periphery. A PRRSv specific proliferation of $\mathrm{CD}^{+} \mathrm{T}$ cells would likely not occur as early as $2 \mathrm{dpi}$, but a bystander proliferation of $\mathrm{CD}^{+} \mathrm{CD}^{+}$memory cells may be a non-specific effect of PRRSv infection, and therefore cannot be excluded. From all investigated PBMC populations $\mathrm{T}$ helper cells seem to be most relevant for the fetal outcome after PRRSv infection. Although only a trend, absolute numbers of $\mathrm{T}$ helper cells over time were positively associated with fetal mortality rate. Therefore, $\mathrm{T}$ helper cell counts could be used as a possible indicator of susceptibility to reproductive PRRSv. This has to be confirmed in future experiments.

The fairly high percentage of CTLs expressing SLADR in this study agrees with previous data investigating 
the phenotypic maturation of porcine $\mathrm{NK}$ and $\mathrm{T}$ cell subsets from birth to six months of age. CTLs in newborn piglets are SLA-DR negative and the first phenotypic change occurs around 3 weeks of age with a massive appearance of SLA-DR ${ }^{+}$CTLs [36]. In our study, the percentage of SLA-DR ${ }^{+}$CTLs significantly increased in INOC gilts on 2 dpi. By assuming that SLADR expression in porcine CTL is a result of previous antigen contact, this points towards a higher loss of naïve (i.e. SLA-DR ${ }^{-}$) CTLs from blood circulation. But again a non-specific bystander proliferation of antigen experienced CTLs cannot be excluded. A higher decrease of naïve CTLs further corroborated by the decrease in $\mathrm{CD} 8 \beta$ expression and increase in SLA-DR expression levels (Figure 8E and F) and as such, $C D 8 \beta^{\text {low }}$ SLA-DR ${ }^{\text {high }}$ CTLs display the phenotype of terminally differentiated CTLs [37].

In conclusion, PRRSv infection with NVSL 97-7895 in pregnant gilts caused a massive, acute decrease in total leukocyte counts affecting all major PBMC populations, most severely NK cells and CTLs. For all PBMC subsets except B cells, counts started to rebound by 6 dpi indicating a well-functioning immune cell homeostasis, explaining the very mild to absent clinical signs following PRRSv infection in pregnant gilts. However, immune cells migrate extensively and only a very small proportion of immune cells are present in the blood [38], complicating the interpretation of PBMC analyses. That being said, three leukocyte populations may predict relevant biological outcomes in PRRS-infected pregnant gilts. Absolute numbers of total T cells and $\gamma \delta \mathrm{T}$ cells were negatively associated with PRRSv viral load (AUC of RNA concentration in serum over $21 \mathrm{dpi}$ ). Additionally, absolute numbers of $\mathrm{T}$ helper cells may predict fetal mortality rate. Although many questions regarding the immune responses remain unanswered, these findings provide insight and clues that may help reduce the impact of PRRSv in pregnant gilts.

\section{Abbreviations}

ASFv: African swine fever virus; AUC: Area under the curve; BVDv: Bovine viral diarrhea virus; CSFv: classical swine fever virus; CD: Cluster of differentiation; CTLs: Cytolytic T lymphocytes; dpi: Days post infection; FCM: Flow cytometry; FMO: Fluorescence minus one; INOC: Gilts inoculated with PRRSV; CTRL: gilts that were sham inoculated; ILL: Innate-like lymphocytes; SLA-DR: Major histocompatibility complex class II; MFI: Mean fluorescence intensity; mAbs: Monoclonal antibodies; NK cells: Natural killer cells; NKR: Natural killer receptor; PBMC: Peripheral blood mononuclear cells; PBS: Phosphate buffered saline; PCV2: Porcine circovirus type 2; PRRSV: Porcine reproductive and respiratory syndrome virus; PRv: Pseudorabies virus; GRT-PCR: Quantitative reverse transcription polymerase chain reaction; Sn: Sialoadhesin; SD: Standard deviation; TCR: T cell receptor; TLR: toll like receptor; WBCs: White blood cells.

\section{Competing interests}

The authors declare that they have no competing interests.

\section{Authors' contributions}

The animal experiment and laboratory work were conducted by AL, CA and $J H$. WG, AS, and JKL were involved in overall experimental design and interpretation of data. Statistical analyses were completed by JH and AL. JH was the Principal Investigator. All authors read and approved the final manuscript.

\section{Acknowledgements}

The authors wish to acknowledge the numerous technicians and students from the Western College of Veterinary Medicine, Vaccine and Infectious Disease Organization, Prairie Diagnostic Services, Inc. and the University of Alberta who assisted with this project. We offer special thanks to Natasa Arsic and Philip Griebel for their greatly valued assistance with the flow cytometry, to lan Dohoo for his guidance with statistical analyses, and to Graham Plastow for the overall coordination of the project. Pregnant gilts were provided and bred by Fast Genetics Inc., Spiritwood with management support of Dawn Friesen, Connie Heisler, Donell Wingerter and Benny Mote. Funding for the project was generously provided by grants from Genome Canada and Genome Prairie (grant number 2209-F), with administrative support from Genome Alberta.

\section{Author details}

${ }^{1}$ Department of Large Animal Clinical Sciences, Western College of Veterinary Medicine, University of Saskatchewan, Saskatoon, SK, Canada. ${ }^{2}$ Institute of Immunology, Department of Pathobiology, University of Veterinary Medicine Vienna, Vienna, Austria. ${ }^{3}$ Animal Parasitic Diseases Laboratory, Beltsville Agricultural Research Center, Agricultural Research Service, U.S. Department of Agriculture, Beltsville, MD, USA. ${ }^{4}$ Current address: University Clinic for Swine, Department for Farm Animals and Veterinary Public Health, University of Veterinary Medicine Vienna, Veterinaerplatz 1, 1210 Vienna, Austria.

Received: 30 May 2014 Accepted: 2 December 2014

Published online: 14 December 2014

\section{References}

1. Allende R, Laegreid WW, Kutish GF, Galeota JA, Wills RW, Osorio FA: Porcine reproductive and respiratory syndrome virus: description of persistence in individual pigs upon experimental infection. J Virol 2000, 74:10834-10837.

2. Wills RW, Doster AR, Galeota JA, Sur JH, Osorio FA: Duration of infection and proportion of pigs persistently infected with porcine reproductive and respiratory syndrome virus. J Clin Microbiol 2003, 41:58-62.

3. Mateu E, Diaz I: The challenge of PRRS immunology. Vet J 2008, 177:345-351.

4. Nauwynck HJ, Van Gorp H, Vanhee M, Karniychuk U, Geldhof M, Cao A, Verbeeck M, Van Breedam W: Micro-dissecting the pathogenesis and immune response of PRRSV Infection paves the way for more efficient PRRSV vaccines. Transbound Emerg Dis 2012, 59:50-54.

5. Yoo D, Song C, Sun Y, Du Y, Kim O, Liu HC: Modulation of host cell responses and evasion strategies for porcine reproductive and respiratory syndrome virus. Virus Res 2010, 154:48-60.

6. Nielsen J, Botner A, Tingstedt JE, Aasted B, Johnsen CK, Riber U, Lind P: In utero infection with porcine reproductive and respiratory syndrome virus modulates leukocyte subpopulations in peripheral blood and bronchoalveolar fluid of surviving piglets. Vet Immunol Immunopathol 2003, 93:135-151.

7. Christianson WT, Choi CS, Collins JE, Molitor TW, Morrison RB, Joo HS: Pathogenesis of porcine reproductive and respiratory syndrome virus infection in mid-gestation sows and fetuses. Can J Vet Res 1993, 57:262-268.

8. Ladinig A, Wilkinson J, Ashley C, Detmer SE, Lunney JK, Plastow G, Harding JC: Variation in Fetal outcome, viral load and ORF5 sequence mutations in a large scale study of phenotypic responses to late gestation exposure to type 2 porcine reproductive and respiratory syndrome virus. PLoS One 2014, 9:e96104.

9. Roederer M: Spectral compensation for flow cytometry: visualization artifacts, limitations, and caveats. Cytometry 2001, 45:194-205.

10. Sinkora M, Butler JE: The ontogeny of the porcine immune system. Dev Comp Immunol 2009, 33:273-283.

11. Yang H, Parkhouse RM, Wileman T: Monoclonal antibodies that identify the CD3 molecules expressed specifically at the surface of porcine gammadelta-T cells. Immunology 2005, 115:189-196.

12. Karalyan Z, Zakaryan H, Arzumanyan H, Sargsyan K, Voskanyan H, Hakobyan L, Abroyan L, Avetisyan A, Karalova E: Pathology of porcine peripheral white blood cells during infection with African swine fever virus. BMC Vet Res 2012, 8:18. 
13. Summerfield A, Knotig SM, McCullough KC: Lymphocyte apoptosis during classical swine fever: implication of activation-induced cell death. J Virol 1998, 72:1853-1861.

14. Page GR, Wang FI, Hahn EC: Interaction of pseudorabies virus with porcine peripheral blood lymphocytes. J Leukoc Biol 1992, 52:441-448.

15. Nielsen J, Vincent IE, Botner A, Ladekaer-Mikkelsen AS, Allan G, Summerfield A, McCullough KC: Association of lymphopenia with porcine circovirus type 2 induced postweaning multisystemic wasting syndrome (PMWS). Vet Immunol Immunopathol 2003, 92:97-111.

16. Pomorska-Mol M, Markowska-Daniel I, Kwit K: Immune and acute phase response in pigs experimentally infected with $\mathrm{H} 1 \mathrm{~N} 2$ swine influenza virus. FEMS Immunol Med Microbiol 2012, 66:334-342.

17. Labarque G, Van Gucht S, Nauwynck H, Van Reeth K, Pensaert M: Apoptosis in the lungs of pigs infected with porcine reproductive and respiratory syndrome virus and associations with the production of apoptogenic cytokines. Vet Res 2003, 34:249-260.

18. Rossow KD, Bautista EM, Goyal SM, Molitor TW, Murtaugh MP, Morrison RB, Benfield DA, Collins JE: Experimental porcine reproductive and respiratory syndrome virus infection in one-, four-, and 10-week-old pigs. J Vet Diagn Invest 1994, 6:3-12.

19. Rossow KD, Collins JE, Goyal SM, Nelson EA, Christopher-Hennings J, Benfield DA: Pathogenesis of porcine reproductive and respiratory syndrome virus infection in gnotobiotic pigs. Vet Pathol 1995, 32:361-373.

20. Sur $J H$, Doster AR, Osorio FA: Apoptosis induced in vivo during acute infection by porcine reproductive and respiratory syndrome virus. Vet Pathol 1998, 35:506-514.

21. Costers S, Lefebvre DJ, Delputte PL, Nauwynck HJ: Porcine reproductive and respiratory syndrome virus modulates apoptosis during replication in alveolar macrophages. Arch Virol 2008, 153:1453-1465.

22. Li Y, Wang G, Liu Y, Tu Y, He Y, Wang Z, Han Z, Li L, Li A, Tao Y, Cai X: Identification of apoptotic cells in the thymus of piglets infected with highly pathogenic porcine reproductive and respiratory syndrome virus. Virus Res 2014, 189:29-33.

23. Wang G, He Y, Tu Y, Liu Y, Zhou EM, Han Z, Jiang C, Wang S, Shi W, Cai X: Comparative analysis of apoptotic changes in peripheral immune organs and lungs following experimental infection of piglets with highly pathogenic and classical porcine reproductive and respiratory syndrome virus. Virol J 2014, 11:2

24. Mueller SN, Gebhardt T, Carbone FR, Heath WR: Memory T cell subsets, migration patterns, and tissue residence. Annu Rev Immunol 2013, 31:137-161.

25. Cahill RN, Frost H, Trnka Z: The effects of antigen on the migration of recirculating lymphocytes through single lymph nodes. J Exp Med 1976, 143:870-888.

26. Hall JG, Morris B: The immediate effect of antigens on the cell output of a lymph node. Br J Exp Pathol 1965, 46:450-454.

27. Duan $X$, Nauwynck HJ, Pensaert MB: Virus quantification and identification of cellular targets in the lungs and lymphoid tissues of pigs at different time intervals after inoculation with porcine reproductive and respiratory syndrome virus (PRRSV). Vet Microbiol 1997, 56:9-19.

28. Calvert JG, Slade DE, Shields SL, Jolie R, Mannan RM, Ankenbauer RG, Welch SK: CD163 expression confers susceptibility to porcine reproductive and respiratory syndrome viruses. J Virol 2007, 81:7371-7379.

29. Nauwynck HJ, Duan X, Favoreel HW, Van Oostveldt P, Pensaert MB: Entry of porcine reproductive and respiratory syndrome virus into porcine alveolar macrophages via receptor-mediated endocytosis. J Gen Virol 1999, 80:297-305

30. Dwivedi V, Manickam C, Binjawadagi B, Linhares D, Murtaugh MP, Renukaradhya GJ: Evaluation of immune responses to porcine reproductive and respiratory syndrome virus in pigs during early stage of infection under farm conditions. Virol J 2012, 9:45.

31. Weesendorp E, Morgan S, Stockhofe-Zurwieden N, Popma-De Graaf DJ, Graham SP, Rebel JM: Comparative analysis of immune responses following experimental infection of pigs with European porcine reproductive and respiratory syndrome virus strains of differing virulence. Vet Microbio/ 2013, 163:1-12.

32. Biron CA, Nguyen KB, Pien GC, Cousens LP, Salazar-Mather TP: Natural killer cells in antiviral defense: function and regulation by innate cytokines. Annu Rev Immunol 1999, 17:189-220.

33. Murphy KP: Janeway's Immunobiology. Garland Science, Taylor \& Francis Group, LLC: New York, USA; 2012
34. Saalmuller A, Reddehase MJ, Buhring HJ, Jonjic S, Koszinowski UH: Simultaneous expression of CD4 and CD8 antigens by a substantial proportion of resting porcine T lymphocytes. Eur J Immunol 1987, 17:1297-1301.

35. Saalmuller A, Werner $T$, Fachinger $V$ : T-helper cells from naive to committed. Vet Immunol Immunopathol 2002, 87:137-145.

36. Talker SC, Kaser T, Reutner K, Sedlak C, Mair KH, Koinig H, Graage R, Viehmann M, Klingler E, Ladinig A, Ritzmann M, Saalmüller A, Gerner W: Phenotypic maturation of porcine NK- and T-cell subsets. Dev Comp Immunol 2013, 40:51-68.

37. Reutner K, Leitner J, Essler SE, Witter K, Patzl M, Steinberger P, Saalmuller A, Gerner W: Porcine CD27: identification, expression and functional aspects in lymphocyte subsets in swine. Dev Comp Immunol 2012, 38:321-331.

38. Di Rosa F, Pabst R: The bone marrow: a nest for migratory memory $T$ cells. Trends Immunol 2005, 26:360-366.

doi:10.1186/s13567-014-0128-

Cite this article as: Ladinig et al:: Changes in leukocyte subsets of pregnant gilts experimentally infected with porcine reproductive and respiratory syndrome virus and relationships with viral load and fetal outcome. Veterinary Research 2014 45:128.

\section{Submit your next manuscript to BioMed Central and take full advantage of:}

- Convenient online submission

- Thorough peer review

- No space constraints or color figure charges

- Immediate publication on acceptance

- Inclusion in PubMed, CAS, Scopus and Google Scholar

- Research which is freely available for redistribution 